Article

\title{
Pupil Function in Pseudophakia: Proximal Miosis Behavior and Optical Influence
}

\author{
Elsa Fonseca ${ }^{1,2, *, t, \neq(0)}$, Paulo Fiadeiro ${ }^{1,2, \neq} \odot$, Renato Gomes ${ }^{1}$, Angel Sanchez Trancon ${ }^{3}$, \\ António Baptista 4 (D) and Pedro Serra ${ }^{3,5, \ddagger(\mathbb{D}}$ \\ 1 Departamento de Física, Universidade da Beira Interior (UBI), Av. Marquês de Ávila e Bolama, \\ 6201-001 Covilhã, Portugal; fiadeiro@ubi.pt (P.F.); renato-andre@hotmail.com (R.G.) \\ 2 Fiber Materials and Environmental Technologies (FibEnTech-UBI), Av. Marquês de Ávila e Bolama, \\ 6201-001 Covilhã, Portugal \\ 3 Cataract and Refractive Surgery Unit, Ophthalmic Clinic Vista Sanchez Trancon, Calle la Violeta, \\ 06010 Badajoz, Spain; sancheztrancon@sancheztrancon.com (A.S.T.); \\ pedro.serra@vistasancheztrancon.com (P.S.) \\ 4 Center of Physics, School of Sciences, University of Minho, Campus de Gualtar, 4710-057 Braga, Portugal; \\ abaptista@fisica.uminho.pt \\ 5 Optics and Optometry Department, Instituto Superior de Educação e Ciência, Alameda das Linhas de \\ Torres, 1750-142 Lisboa, Portugal \\ * Correspondence: efonseca@ubi.pt \\ $+\quad$ This paper is an extended version of our paper published in 4th International Conference on Applications of \\ Optics and Photonics, Costa MF, Coelho JMP, Cabral A. (Eds); SPOF, 2019. \\ $\ddagger$ These authors contributed equally to this work.
}

Received: 25 September 2019; Accepted: 1 November 2019; Published: 6 November 2019

\begin{abstract}
The pseudophakic eye lacks the ability to produce a refractive change in response to object proximity. Thus, individual anatomical features such as the pupil size play an important role in achieving functional vision levels. In this work, the range of pupil sizes at varying object distance was measured in pseudophakic participants. Furthermore, the impact of the measured values on eye optical quality was investigated using a computer simulation model. A binocular eye-tracker was used to measure the participants' pupil sizes at six object distances, ranging from $0.33 \mathrm{~m}$ (i.e., vergence of $3.00 \mathrm{D}$ ) to $3.00 \mathrm{~m}$ (i.e., vergence of $0.33 \mathrm{D}$ ), while observing a Maltese cross with a constant angular size of $1^{\circ}$. In total, 58 pseudophakic participants were enrolled in this study (age mean \pm standard deviation: $70.5 \pm 11.3$ years). The effects of object distance and age on pupil size variation were investigated using linear mixed effects regression models. Age was found to have a small contribution to individual variability. The mean infinite distance pupil size (intercept) was 4.45 (95\% CI: $2.74,6.17) \mathrm{mm}$ and the mean proximal miosis (slope) was -0.23 (95\% CI: $-0.53,0.08) \mathrm{mm} / \mathrm{D}$. The visual acuity (VA) estimation for a distant object ranged from -0.1 logMAR (smallest pupil) to $0.04 \log$ MAR (largest pupil) and the near VA $(0.33 \mathrm{~m})$ when mean proximal miosis was considered ranged from $0.28 \log$ MAR (smallest pupil) to $0.42 \log$ MAR (largest pupil). When mean distance pupil was considered, proximal miosis individual variability produced a variation of $0.04 \operatorname{logMAR}$ for the near object and negligible variation for the distant object. These results support the importance of distance pupil size measurement for the prediction of visual performance in pseudophakia, while suggesting that proximal miosis has a negligible impact in VA variability.
\end{abstract}

Keywords: pupil size; cataract surgery; pseudophakic; ocular accommodation; visual assessment; visual optics; optical quality; eye model 


\section{Introduction}

The presence of a near object causes the eyes to converge, increases the dioptric power by means of changes in the crystalline lens radius of curvature and decreases the pupil size through proximal miosis [1]. With the implantation of an intraocular lens (IOL), the pseudophakic eye is still capable of eliciting this accommodative mechanism, as documented by a reduction in the ciliary muscle ring [2], the ability to converge, and the presence of pupil miosis. The compression induced by the ciliary muscle ring on the IOL haptics produces neither a constant displacement of the IOL $[3,4]$ nor a significant movement able to generate a functional refractive change $[5,6]$. Therefore, the pseudophakic eye implanted with a monofocal IOL is unable to increase its dioptric power in order to focus near objects.

Despite this fact, there is clinical evidence that a percentage of pseudophakic eyes can attain functional levels of near vision [7]. This ability, named pseudoaccommodation, describes the dioptric interval in which an object can be observed under a certain level of defocus [8]. Several anatomical and optical features were associated with increased pseudoaccommodation, such as pupil diameter [9], anterior chamber depth [10], age [11], post-operative astigmatism [12], cornea [13] and ocular higher order aberrations [14], and corneal multifocality [15]. Recently, it has been shown that pupil diameter was the main predictor for increased pseudoaccommodation [16] and near visual acuity, as well as reading performance [17]. These findings corroborate the theoretical role of pupil diameter in determining the retinal blur area and, consequently, the depth-of-field [18].

Pupil diameter is highly variable among the population, with the variability being associated with several intraindividual features (see [19], for a brief review). For instance, in a phakic population aged above 60 years old, i.e., age-matched with normal pseudophakic population, distance pupil size may span the 2.0-7.0 mm interval, showing some narrowing for higher illumination levels [20]. Using optical modeling, a near emmetropic pseudophakic eye with a monofocal LIO decreased its optical quality by nearly $40 \%$ with a variation in pupil size from 2.0 to $5.0 \mathrm{~mm}$ [21]. This effect may be stronger in the presence of defocus due to the lack of refractive change when a near stimulus is applied.

Studies assessing the effect of pupil size in pseudophakic visual performance tend to use a single measure of the pupil size performed monocularly with a pupillometer [22]. There are some important limitations in this approach with respect to visual assessment conditions since it accounts for neither binocularity nor object distance [23,24]. Recently, Almutairi et al. [24] reported an average pupil decrease of $0.24 \mathrm{~mm} / \mathrm{D}$ of stimulus vergence, in different age groups, including full presbyopes. This finding contrasts with a previous study reporting no systematic variation in proximal miosis in a similar population when measured in monocular viewing conditions, adding evidence to the importance of convergence as a driver for proximal pupil miosis. Adding this fact to the smaller pupil size observed under monocular viewing conditions compared to the most common binocular conditions [25], suggests that pupil size should be assessed under more natural conditions [26]. Furthermore, to our knowledge, there is no available formula, in the literature, describing the pupil diameter behavior of pseudophakic eyes for a range of object distances, as previously described for phakic subjects $[27,28]$.

In this study, the pupil size of pseudophakic participants under binocular vision was measured at various object distances. The measured pupil sizes were used to generate two models to describe the pupil diameter variation with object distance. A simple model using object vergence to model pupil size [29] is compared to a more elaborated model including age as a variable. The model was further applied in an optical model to estimate the magnitude of the effect of intraindividual variations, individual pupil size and pupil proximal miosis, on Visual Acuity (VA).

\section{Materials and Methods}

\subsection{Participants}

Patients attending a routine eye exam in the Ophthalmology Clinic Vista Sanchez Trancon (Badajoz, Spain) were selected for the study. All participants had implanted bilaterally a monofocal 
spherical intraocular lens (RayOne Spheric, Rayner Intraocular Lenses Limited, West Sussex, UK) by the same surgeon. The exclusion criteria were as follows: eventful cataract surgery, presence of ocular media or retinal anomalies, abnormal pupil shape, unresponsive pupil to light reflex and post-operative refractive error expressed in spherical equivalent higher than \pm 1.00 DS. Subjective refraction had as starting point the objective refraction (Topcon KR-8900, Topcon Corporation, Tokyo, Japan); the cylinder component was refined using the Jackson-Cross Cylinder technique and the spherical component using \pm 0.25 DS lenses. All participants gave their written informed consent to take part in the study. The research had the ethical approval from the University of Beira Interior Faculty of Health Sciences Ethics Committee.

\subsection{Pupil Size Measurement}

The participants pupils' diameters were recorded using a binocular eye-tracker (Arrington Research, Scottsdale, AZ, USA, sampling rate $220 \mathrm{~Hz}$ ) controlled by a custom algorithm written in MATLAB ${ }^{\circledR}($ R2016b, MathWorks, MA, USA). The participants looked at a white circle (with angular size of $5.5^{\circ}$ and luminance of $65.3 \mathrm{~cd} \mathrm{~m}^{-2}$ ) displayed on an LCD monitor (LG model 23MP65HQ), with a Maltese cross (angular size: $1.0^{\circ}$ ) on its center used as a fixation point. The object distances tested, given by the monitor position, were $3.0,1.0,0.66,0.50,0.40$ and $0.33 \mathrm{~m}$. The stimulus angular size was kept constant for the given distances. The amount of light reaching the corneal plane was 0.45 lux (luxmeter Minolta, model T-10A, Konica Minolta, Europe) and was also kept constant for the different object positions. The pupil diameters were measured from the furthest $(3.0 \mathrm{~m})$ to the closest $(0.33 \mathrm{~m})$ object position. The participants sat in a dark room with the head on a headrest. Initially, the participants had $2 \mathrm{~m}$ of light adaptation period staring at the white circle positioned at $3.0 \mathrm{~m}$, and then, for each object position, there was a $5 \mathrm{~s}$ light adaptation period followed by $10 \mathrm{~s}$ of pupil diameter measurement. Prior to the measurements, each participant was instructed to focus the Maltese cross and blink normally. For each object position, the pupil diameter was calculated by removing the blinks from the sampled data, using a Hampel filter, followed by averaging of the sampled data. An example of pupil size acquisition during $15 \mathrm{~s}$ ( $5 \mathrm{~s}$ light adaptation plus $10 \mathrm{~s}$ test) is presented in Figure 1, where both the raw data and the processed data for blink removal are displayed.
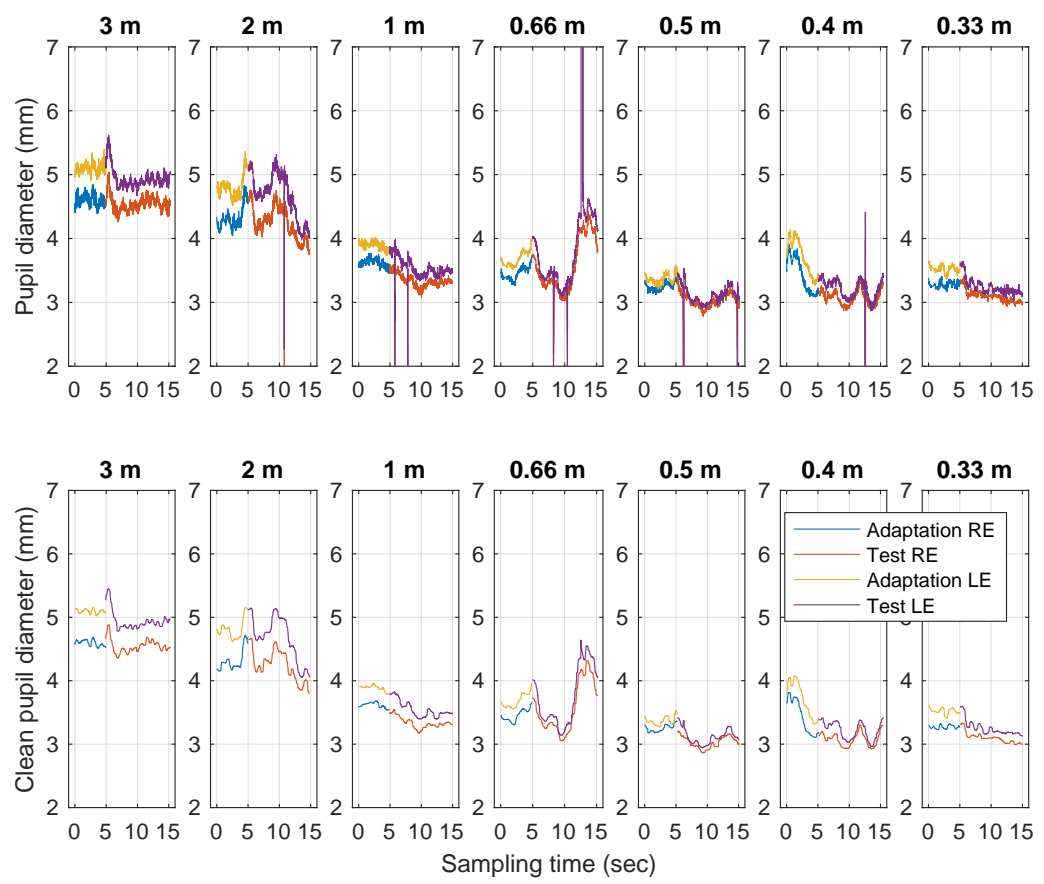

Figure 1. Pupil diameter recordings during $15 \mathrm{~s}$ and for the different observing distances. Upper set: The raw data for the RE and LE. Lower set: The pupil sizes after blink removal for the RE and LE using the Hampel filter. 


\subsection{Pupil Size Statistical Modeling}

The pupil diameter as a function of object position reciprocal, defined as object vergence, was modeled by fitting nonlinear mixed-effects models [30]. Since data taken at each observation distance can be regarded as a repeated measure for each participant, correlations between measurements must be considered in the regression model. Mixed-effects models recognize correlations within sample subgroups and provide a more robust alternative to fitting data separately to each patient, by expressing each model parameters as a sum of a fixed effect and a random effect. Estimating the fixed effects gives a description of the global sample, while estimating the random effects gives a description of specific groups, in this case individual participants, within the data.

In this work, several mixed effect models were investigated, which included different combinations of fixed effects, namely the slope and intercept with respect to object vergence, the slope and intercept with respect to age and a term that accounts for age-vergence interaction. The tested random effects structure included the slope and intercept with respect to object vergence.

The selected model includes the response variable given by the pupil size $y_{i j}$, for the $i$ th participant at the $j$ th object vergence, with the following mathematical expression:

$$
y_{i j}=f\left(\phi_{i}, a_{i}, x_{i j}\right)+\varepsilon_{i j}, \quad i=1, \ldots, N ; \quad j=1, \ldots, M,
$$

where $x_{i j}$ is the value of the predictor variable (object vergence), $a_{i}$ is the individual age, $\phi_{i}$ is the parameter vector that governs the response of the $i$ th participant to the predictor variable, and $\varepsilon_{i j}$ is the measurement error modeled by a normally distributed random variable with zero mean. The number of participants is represented by $N$, the number of object positions is given by $M$, and function $f$ specifies the shape of the model. The parameter vector $\phi_{i}$ accounts for between-participant variation and can be expressed as:

$$
\phi_{i}=A_{i} \beta+B_{i} b_{i} ; \quad b_{i} \sim N(0, D),
$$

where $\beta$ is the vector of fixed effects parameters, $b_{i}$ is the vector of random effects associated with the $i$ th participant, $D$ is the variance-covariance matrix for the random effects, and $A_{i}$ and $B_{i}$ are the design matrices for the fixed and random effects, respectively.

Starting with the investigation of the best response curve, both linear and nonlinear (sigmoidal) shapes were tested using the nonlinear mixed-effects model, since it is the most general approach. For the range of tested object vergences, the linear model yielded the best results. In this case, the linear model is given by:

$$
f\left(\phi_{i}, a_{i}, x_{i j}\right)=\beta_{1}+b_{i 1}+\beta_{2} x_{i j}+b_{i 2} x_{i j}+\beta_{3} a_{i}+\beta_{4} a_{i} x_{i j},
$$

where the non-zero $\beta$ and the $b_{i}$ coefficients are explicitly presented, according to the respective design matrices. The process of mixed-effects model construction and the application of selection criteria for model performance evaluation and significant effects selection follow the methodology described in [31]. The selection of the significant random effects was based on both the Akaike Information Criterion (AIC) [32] and the Bayesian Information Criterion (BIC) [33]. The method of maximum likelihood was used to estimate the fixed effects, while best linear unbiased predictor (BLUP) was used to predict the random effects. The performance of the model was evaluated using Bias, root mean square error (RMSE) and coefficient of determination $R^{2}$. These statistical parameters are defined as follows:

$$
\begin{gathered}
\text { Bias }=\frac{\sum_{j=1}^{M} \sum_{i=1}^{N}\left(y_{i j}-\hat{y}_{i j}\right)}{M N}, \\
\text { RMSE }=\left[\frac{\sum_{j=1}^{M} \sum_{i=1}^{N}\left(y_{i j}-\hat{y}_{i j}\right)^{2}}{(M N-1)}\right],
\end{gathered}
$$




$$
R^{2}=1-\sqrt{\frac{\sum_{j=1}^{M} \sum_{i=1}^{N}\left(y_{i j}-\hat{y}_{i j}\right)^{2}}{\sum_{j=1}^{M} \sum_{i=1}^{N}\left(y_{i j}-\overline{y_{j}}\right)^{2}}},
$$

where $\hat{y}_{i j}$ is the predicted value for the $i$ th participant at the $j$ th object vergence, and $\overline{y_{j}}$ is the mean of the $\overline{y_{j}}$ across the participants.

The regression model was implemented in MATLAB ${ }^{\circledR}(R 2016 b)$ using the function nlmefit from the Statistics and Machine Learning Toolbox, with the option LME (method of maximum likelihood). The fitting routine also provides an estimate of the variance-covariance matrix $D$.

\subsection{Optical Performance Modeling}

The effect of pupil size on visual performance can be, at least in part, theoretically modeled through the relation between its magnitude and the size of the blur area produced in the retina or other image quality metrics. The latter parameter also depends on the paraxial properties and the aberrations of the eye. These can be modeled using a schematic eye model and numerical simulation. Herein, a pseudophakic schematic eye, adapted from the well-known Liou and Brennan (LBME) model [34], was used to predict the visual quality dependence on pupil size. The ray tracing calculations were performed with OSLO Premium 7.0 (Lambda Research Corp., Littleton, MA, USA) optical design software. This model was chosen based on its anatomical, biometric and optical accuracy. Furthermore, several pseudophakic versions of this model have been extensively tested in previous studies regarding IOL optical performance [35]. The LBME comprises four aspheric refractive surfaces, a gradient index lens and an iris pupil with a decentration of $0.50 \mathrm{~mm}$ to the nasal side. The pseudophakic version of the LBME used in this study includes a typical monofocal intraocular lens with a spherical design: the MC5812AS lens (Dr. Schmidt Intraocularlinsen GmbH, St. Augustin, Germany) [35], made of hydrophilic acrylic with a refractive index of 1.461 and a central thickness of $1.057 \mathrm{~mm}$. The IOL has a biconvex configuration with spherical surfaces curvature radius given by $13 \mathrm{~mm}$ and $-10 \mathrm{~mm}$ for the anterior and posterior sides, respectively. Axial placement of the IOL is given by the anterior chamber depth (ACD) value in milimeters:

$$
A C D=-68.747+0.62467 \times A
$$

where $A=118.6$ is the constant, used in the SRK/T formula, yielding an ACD value of $5.35 \mathrm{~mm}$. The pupil was decentered with respect to the optical axis of the eye by $-0.5 \mathrm{~mm}$ to the nasal side and the Stiles-Crawford effect was implemented by means of a Gaussian profile at the entrance pupil. A prescription of the pseudophakic LBME is summarized in Table 1.

Table 1. Prescription details of the pseudophakic Liou and Brennan model eye.

\begin{tabular}{lrrrr}
\hline Surface & Radius $\mathbf{( m \mathbf { m } )}$ & Thickness $\mathbf{( m \mathbf { m } )}$ & Conic Constant & $\boldsymbol{n}(\boldsymbol{\lambda}=\mathbf{5 5 5} \mathbf{n m})$ \\
\hline Anterior cornea & 7.77 & 0.500 & -0.18 & 1.376 \\
Posterior Cornea & 6.40 & 3.160 & -0.60 & 1.336 \\
Iris & $\infty$ & 1.512 & - & 1.336 \\
Anterior IOL & 13.00 & 1.057 & - & 1.461 \\
Posterior IOL & -10.00 & 17.721 & - & 1.336 \\
Retina & -12.00 & 0.000 & - & - \\
\hline
\end{tabular}

The visual quality simulations were conducted for the same object distances as the ones used in the pupil measurement experiment. The initial rest state was set to infinity in the computer eye model to simulate distant vision as the paraxial case. Thus, it was considered that the best distant correction was obtained under this approximation. According to this rationale, the optical performance of the model eye was optimized for a distant target. This was done by maximizing the modulation transfer 
function based visual Strehl ratio (VSMTF) with respect to the vitreous length, since, according to Thibos et al. [36], this image plane metrics is well correlated with the best subjective VA.

$$
\operatorname{VSMTF}=\frac{\iint_{-\infty}^{\infty} \operatorname{CSF}_{\mathrm{N}}\left(f_{x}, f_{y}\right) \operatorname{MTF}\left(f_{x}, f_{y}\right) d f_{x} d f_{y}}{\iint_{-\infty}^{\infty} \operatorname{CSF}_{\mathrm{N}}\left(f_{x}, f_{y}\right) \operatorname{MTF}_{\mathrm{DL}}\left(f_{x}, f_{y}\right) d f_{x} d f_{y}}
$$

where $\mathrm{CSF}_{\mathrm{N}}$ is the neural contrast sensitivity function [37], the subscript DL stands for diffraction limited, and the MTF through frequency curves were obtained from the arithmetic mean of the respective sagittal and tangential sections measured on-axis. To investigate the optical performance predicted by the eye model, the area under the modulation transfer function (aMTF), for spatial frequencies up 50 cycles/mm, was computed according to:

$$
\mathrm{aMTF}=\sum_{f=1}^{50} \operatorname{MTF}(f) / 50 .
$$

This metric has a high correlation with the clinical through-focus VA measured in pseudophakic patients, as has been shown in recent study [38] using an optical bench eye model. Using the results from this work, the aMTF values can be converted into VA values (logMAR) using the following linear regression equation:

$$
V A(\log \mathrm{MAR})=-0.2038+0.0775 \times \mathrm{aMTF}^{-1} .
$$

The computation of the VSMTF and the aMTF metrics for each object distance and corresponding pupil size was performed in MATLAB ${ }^{\circledR}$ (R2016b). Before performing the through focus computation of the VSMTF and the aMTF values, it was necessary to optimize the eye model, using the ray tracing software implementation of the LBME, to resemble a distance corrected pseudophakic eye. First, the object was set to the distant position (infinity) and the pupil size was selected as the mean far distance pupil obtained from the experimental measurements. After retrieving the set of Zernike coefficients, using the Zernike Analysis of Wavefront feature of OSLO Premium, and convert it to the OSA standard version [39], the $\operatorname{MTF}\left(f_{x}, f_{y}\right)$ and $\operatorname{MTF}_{\mathrm{DL}}\left(f_{x}, f_{y}\right)$ functions were generated for a set of defocus values. Then, using Equation (8), the VSMTF values were computed from these functions. The defocus value that maximizes the VSMTF was retrieved and the corresponding vitreous length was computed.

Finally, the aMTF curves were used to generate depth of focus estimates. The estimated depth of focus (DOF) is the range of object vergences (in diopters) over which the estimated VA is 0.2 logMAR or better [40]. Other studies have investigated the effect of pupil miosis on depth of focus of presbyopic or pseudophakic eyes using the optical transfer function based visual Strehl ratio (VSOTF) [41]. However, they used a different simulation methodology, based on typical zernike aberrations and through focus analysis [42], followed by a criterion based on the peak of the VSOTF to compute the DOF.

\section{Results}

The study comprised 116 eyes of 58 participants with mean age $70.5 \pm 11.3$ years (range: 43-90 years). The comparison between the right and the left eyes, using two-way repeated measures ANOVA, showed no statistical difference between the right eye (RE) and the left eye (LE) $(F(1.58)=1.929, p=0.170)$. Moreover, no significant interaction between eye and object position was found $(F(5.290)=0.285, p=0.921)$, indicating that the two eyes had similar pupil sizes, and both reacted equally to the object position. Therefore, only the pupil size from the RE was used for analysis. The mean subjective spherical equivalent for the RE was $-0.15 \pm 0.29 \mathrm{D}$. Furthermore, no association was found between maximum pupil size and mean subjective spherical equivalent (Pearson: $R=0.0005, p=0.98$ ), as can be inferred from Figure 2 . 

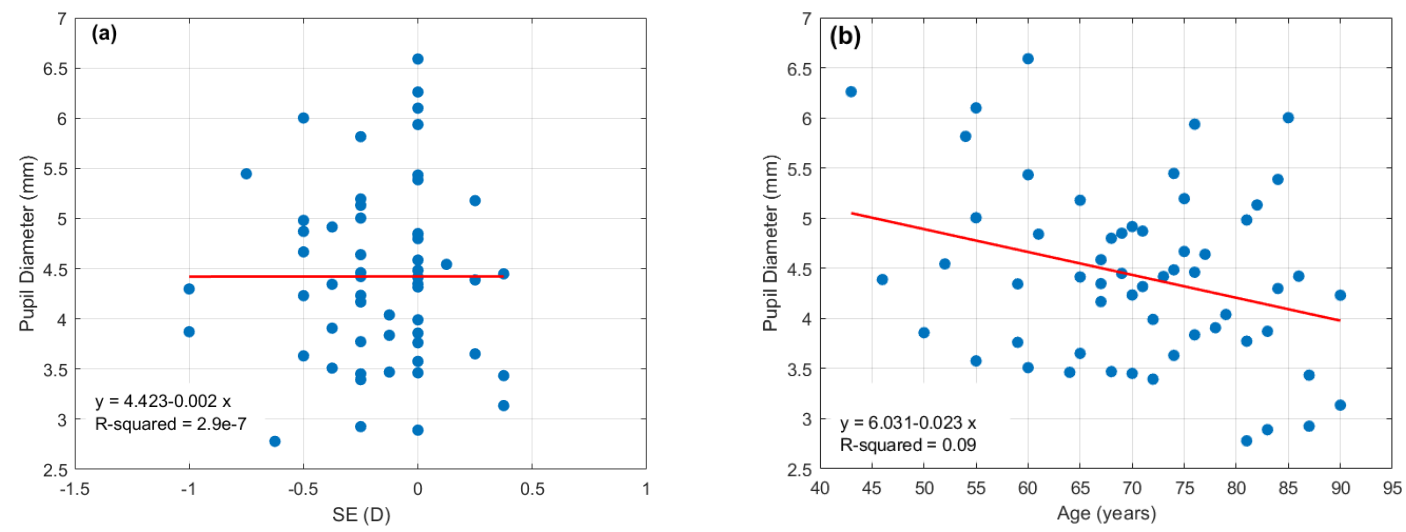

Figure 2. (a) Far distance $(3.0 \mathrm{~m})$ pupil diameter versus subjective spherical equivalent. (b) Far distance $(3.0 \mathrm{~m})$ pupil diameter versus age.

\subsection{Pupillary near Response}

The mean and standard deviation (SD) of the individual pupil diameter values measured at each object vergence are presented in Table 2. These statistical values do not take into account the regression model yet. The mean pupil diameter decreased with increasing object vergence $(F(5.290)=72.013, p<0.0001)$. A significant correlation was found between maximum pupil size and age (Pearson: $R=-0.27, p=0.047$ ), representing a $-0.23 \mathrm{~mm}$ reduction in distance pupil diameter per decade of life. No significant correlations were found between age and pupil proximal miosis (Spearman: $R=0.22, p=0.090$ ) or pupil proximal miosis and maximum pupil size (Spearman: $R=-0.18, p=0.19$ ).

Table 2. Pupil diameter as a function of object vergence (mean $\pm \mathrm{SD}$ ).

\begin{tabular}{llllllll}
\hline & \multicolumn{6}{c}{ Object Vergence (D) } \\
\hline & & 0.33 & 1.00 & 1.50 & 2.00 & 2.50 & 3.00 \\
\hline $\begin{array}{l}\text { Pupil Size } \\
(\mathrm{mm})\end{array}$ & Mean & 4.44 & 4.24 & 4.10 & 3.99 & 3.91 & 3.84 \\
\cline { 2 - 8 } & SD & 0.87 & 0.90 & 0.88 & 0.87 & 0.86 & 0.86 \\
\hline
\end{tabular}

Two linear mixed effects models are presented for comparison. The first model is simpler and considers the pupil size variation as function of object vergence only. This amounts to neglecting the fixed effects $\beta_{3}$ and $\beta_{4}$ of Equation (3), corresponding to the slopes of age and interaction of age and object vergenge, respectively. The second model adds these coefficients, in accordance to Equation (3). The reason for introducing this slightly more complex model is related to the well-known age dependence of the far pupil size and its statistical significance in the present study, so it would be relevant to investigate how much of the inter-individual variability would be explained by this factor.

Table 3 presents the fixed effects parameter estimates (mean and standard error of the mean), the predicted variance-covariance matrix components (for random effects) and the fit statistics for the first and the second mixed models. For the first linear mixed effects model, the pupil size variation as function of object vergence includes the fixed effects described by the following equation:

$$
P D(\mathrm{~mm})=4.451(\mathrm{~mm})-0.227(\mathrm{~mm} / \mathrm{D}) \times O V(\mathrm{D}),
$$

where $P D$ denotes the pupil diameter in milimeters and $O V$ expresses the object vergence in diopters. This model is depicted in Figure 3, on the left plot, along with the confidence levels. For the far distance pupil diameter, a $95 \%$ confidence interval of $[2.74,6.17] \mathrm{mm}$ was obtained, whereas, for the pupil miosis, a $[-0.53,0.08] \mathrm{mm} / \mathrm{D}$ was obtained. Figure 3 also shows the individual residuals that were normally distributed around zero. 

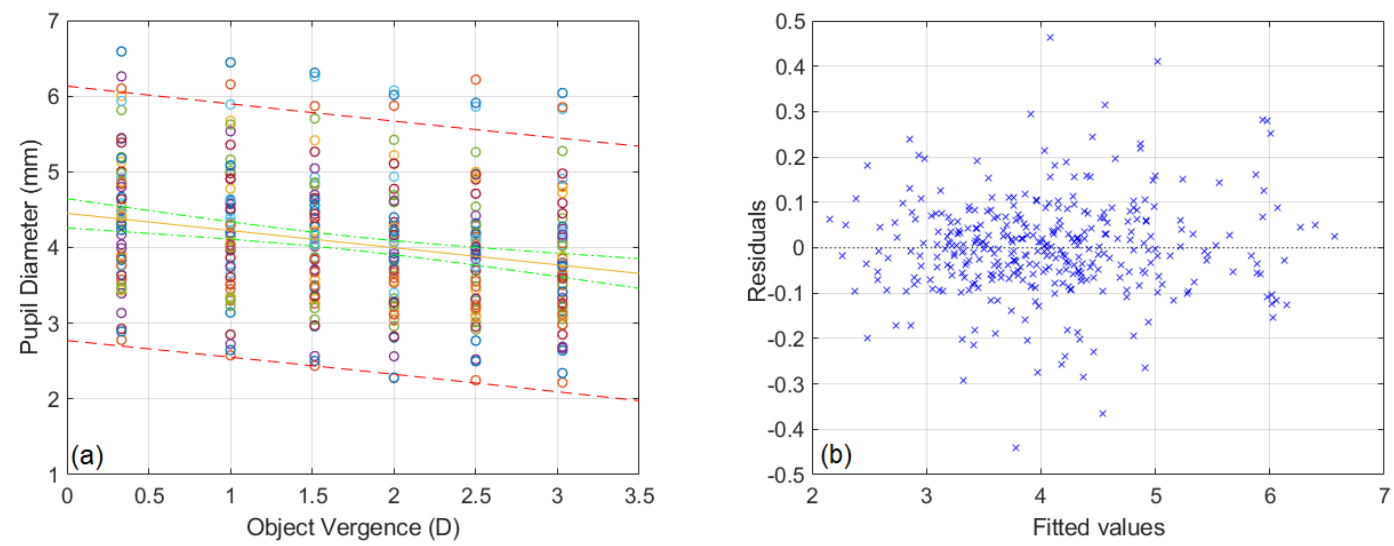

Figure 3. (a) Fixed effect fit and 95\% confidence limits for the mean (dashed green line) and for a single prediction (dashed red line) for a given value of pupil diameter. (b) Scatter plot of standardized residuals versus fitted values for the first mixed effects model.

For the second model, the pupil size as function of age and vergence includes the fixed effects described by

$$
\begin{aligned}
P D(\mathrm{~mm})=5.971(\mathrm{~mm})- & 0.496(\mathrm{~mm} / \mathrm{D}) \times O V(\mathrm{D}) \\
& -0.022(\mathrm{~mm} / \mathrm{y}) \times a(\mathrm{y})+0.004[\mathrm{~mm} /(\mathrm{y} . \mathrm{D})] \times a(\mathrm{y}) \times O V(\mathrm{D}),
\end{aligned}
$$

where $a$ denotes the age in years (y). Table 4 shows the results of the comparison of the two models using the Likelihood Ratio Test (LRT). Both information criteria are presented, with a reduction of the AIC for the age dependent model, but an increase of the BIC in the latter case as a consequence of the increase of the number of model parameters (increment of degrees of freedom by 2 units). It is indicated that Models 1 and 2 are significant different $(\mathrm{LRT}=6.022, p=0.049)$.

Table 3. Estimated parameters and fit statistics for the proposed mixed effects models. The fixed effects parameter estimates are given by mean (standard error).

\begin{tabular}{llcr}
\hline & Parameter & First Model & Second Model \\
\hline \multirow{4}{*}{ Fixed } & $\beta_{1}$ & $4.451(0.117)$ & $5.971(0.725)$ \\
parameters & $\beta_{2}$ & $-0.227(0.022)$ & $-0.496(0.136)$ \\
& $\beta_{3}$ & - & $-0.022(0.010)$ \\
& $\beta_{4}$ & - & $0.004(0.002)$ \\
\hline \multirow{2}{*}{ Variance } & $\sigma_{b_{1}}^{2}$ & 0.789 & 0.732 \\
components & $\sigma_{b_{2}}^{2}$ & 0.025 & 0.023 \\
& $\sigma_{b_{1} b_{2}}$ & -0.052 & -0.042 \\
\hline \multirow{2}{*}{ Goodness } & Bias & 0.000 & 0.000 \\
-of-fit & $R M S E$ & 0.129 & 0.129 \\
& $R^{2}$ & 0.985 & 0.985 \\
\hline
\end{tabular}

Table 4. Mixed effect model performance comparison.

\begin{tabular}{lllllll}
\hline Model & DF & AIC & BIC & Loglikelihood & LRT & $p$-Value \\
\hline 1 & 6 & 16.723 & 39.837 & -2.362 & & \\
2 & 8 & 14.702 & 45.519 & 0.649 & 6.0217 & 0.0493 \\
\hline
\end{tabular}

Both models are good descriptors of the pupil size variation with the object vergence, as given by an $R^{2}$ of 0.985 in both models. The factor age and its influence on the pupil variation with vergence has a minimal effect on pupil variation model with object position. 
For the range of age values obtained in the present study, the inter individual variability seems to play a much more important role in range of observed pupil size values than age variation.

\subsection{Optical Performance}

The previous linear mixed effects model was used to study the impact of far distance pupil diameter and pupil miosis on the retinal image quality of a pseudophakic emmetropic eye. To simulate the average, upper and lower limiting conditions, for the effect of pupil size and proximal miosis, five implementations of the LBME were constructed.

The five implementations of the eye model, which generated five pupil variation curves, were simulated using two sets of parameters: in Case 1, a constant pupil miosis defined as $-0.23 \mathrm{~mm} / \mathrm{D}$ and a variable distance pupil $(2.74,4.45,6.17) \mathrm{mm}$ was considered, while, in Case 2 , a variable miosis $(-0.53,-0.23,-0.04) \mathrm{mm} / \mathrm{D}$ was combined with a constant distance pupil of $4.45 \mathrm{~mm}$. Note that for the lowest level of miosis $(-0.04 \mathrm{~mm} / \mathrm{D})$ the smallest observed value was used instead of the calculated positive value, since we consider that a mydriasis, no matter how small it may be, would not make sense in this case.

Figure 4 presents the area under MTF curves for the emmetropic LBME, from near $(O V=-3.00 \mathrm{D})$ to far $(O V=0 \mathrm{D})$ stimulus distance, from which the AV curves can be estimated, according to Equation (10). It can be seen that, for the mean distance pupil and mean pupil miosis conditions, the retinal image optical quality improves almost linearly from intermediate range to far distance stimuli (from approximately $-2 \mathrm{D}$ or $-0.50 \mathrm{~m}$ to infinity). For larger pupils, however, the plot suggests a stronger exponential-like improvement on the far side range.
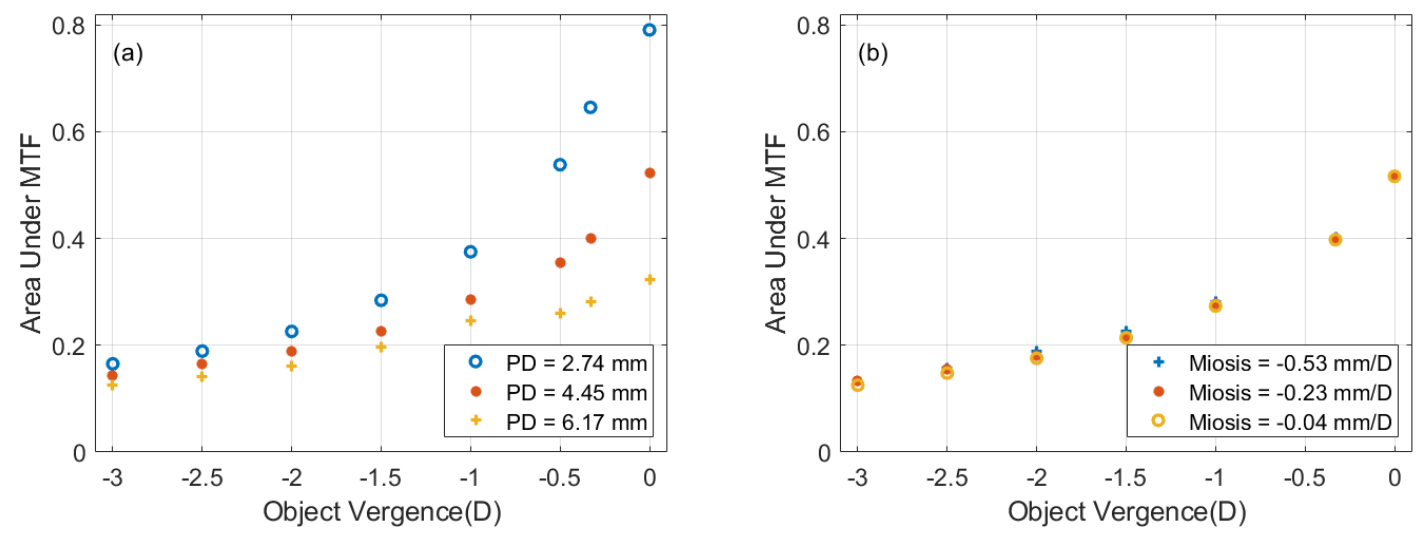

Figure 4. Area under MTF as a function of object vergence in diopters, from near $(O V=-3 \mathrm{D})$ to far $(O V=0 \mathrm{D})$ object distances: (a) Case 1 models with constant pupil miosis and varying distance pupil diameter (PD) values; and (b) Case 2 models with constant distance pupil and varying pupil miosis.

Due to the non-linear relationship between the predicted VA, given by Equation (10), and the area under MTF, the above-mentioned findings can be interpreted from a different perspective. Figure 5 presents the variation of predicted visual acuity in logMAR (MAR: Minimum Angle of resolution) from near $(O V=-3.00 \mathrm{D})$ to far $(O V=0 \mathrm{D})$ object distances. Figure 5 a shows a reduction in visual acuity (logMAR) as the object approaches the eye. Smaller pupils yield higher retinal image quality and, therefore, better VA, with the differences increasing with the amount of defocus. For an object positioned at infinity, smaller pupils have a predicted VA of $-0.1 \log$ MAR, whereas pupils in the upper $95 \% \mathrm{CI}$ limit have a predicted VA of $0.04 \log$ MAR. When the object is positioned at $0.33 \mathrm{~m}$ (i.e., at $-3.00 \mathrm{D}$ ), the VA reduces to $0.27 \log \mathrm{MAR}$ and to $0.42 \log \mathrm{MAR}$ for the smallest and largest pupils, respectively. This means that individuals with larger pupils require an object (a character's height) about $1.5 \times$ bigger to be able to discriminate it. 

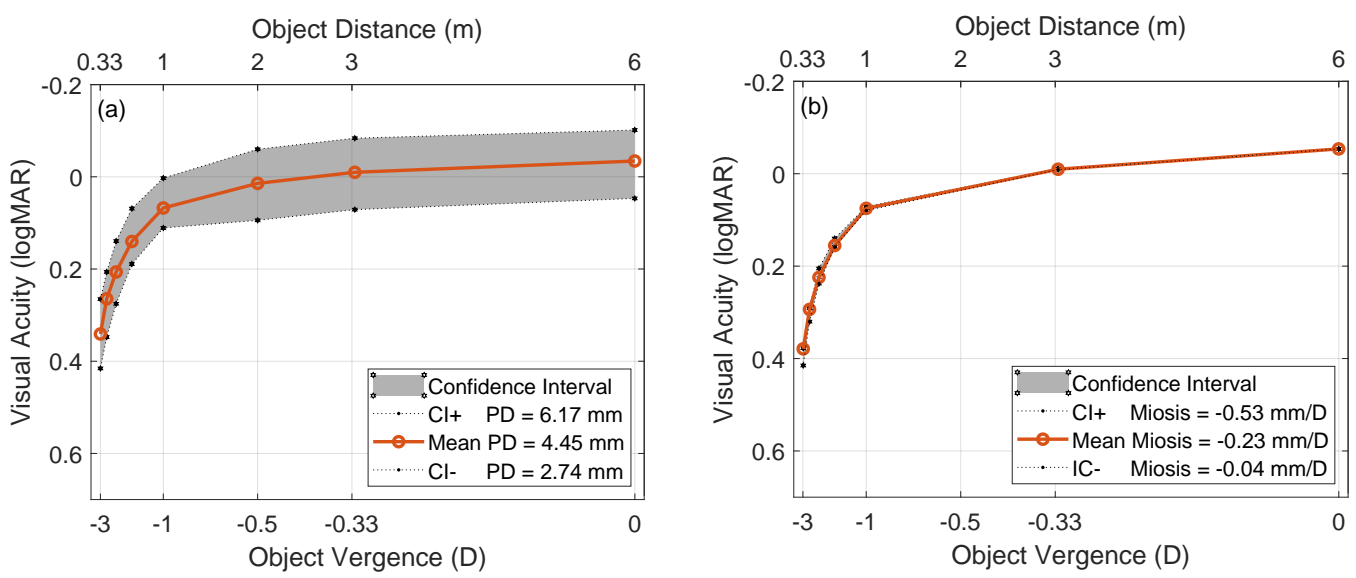

Figure 5. Predicted visual acuity in logMAR (MAR: Minimum Angle of resolution) variation as function of object vergence in diopters, using the simulated area under MTF curve and Equation (10), from near $(O V=-3 \mathrm{D})$ to far $(O V=0 \mathrm{D})$ object distances: (a) Case 1 models with constant pupil miosis and varying distance pupil diameter (PD) values; and (b) Case 2 models with constant distance pupil and varying pupil miosis.

In Figure 5b, the VA predictions for the effect of proximal miosis variation with a constant distance pupil size $(4.45 \mathrm{~mm})$ show similar levels of VA degradation. Although the pupil miosis observed ranged from -0.04 to $-0.53 \mathrm{~mm} / \mathrm{D}$, it produced a minimal variation in VA (0.00 logMAR to $0.04 \log$ MAR, for a distant object and for a near object, respectively).

The predicted VA curves allow estimating the DOF values for each distance pupil diameter model, as previously described. The minimum, mean and maximum distant pupils yield DOF estimates of $2.45 \mathrm{D}, 1.95 \mathrm{D}$ and $1.50 \mathrm{D}$, respectively.

\section{Discussion}

Pupil size is a relevant contributor to optical performance of the eye as it modulates the effect of optical aberrations and depth of field extension with influence in the visual performance of pseudophakic eyes [16]. This study assessed and modeled the pupil size of pseudophakic participants as a function of object position. Additionally, the influence of pupil size and its dynamics on the optical (visual) quality of the pseudophakic eye was determined using optical simulations. The main findings show a large inter-individual variability in the pupil size, and various rates of pupil proximal miosis, demonstrating the presence of pupil dynamics and response to proximal objects in individuals with absence of functional accommodation. The optical simulations indicate that inter-individual pupil differences account for clinical significant differences in the predicted VA. Regarding proximal miosis, inter-individual variability does not produce clinical significant variations in the predicted VA.

The pupil size recording system used provided both pupil measurements under binocular vision in natural but controlled light conditions. The average pupil size for a target distance of $3.0 \mathrm{~m}$ $(4.45 \pm 0.87 \mathrm{~mm})$ compares with the pupil size of presbyopes when measured in similar viewing conditions $(4.70 \pm 0.85 \mathrm{~mm})$ [2]. The present data also show a large inter-individual variability in pupil size spanning from approximately $3.0 \mathrm{~mm}$ to $6.0 \mathrm{~mm}$, similar to those previously reported $[2,20]$. This variability represents the individuals' response to light and the influence of various factors such as mental activity [43] and emotional state [44], which were not controlled. However cognitive effects have a small effect on pupil size [28]. Other more important factors such as accommodation [45], stimulus contrast [46], luminance [20], retinal eccentricity [47], field size [48] and refractive error [49] were controlled in the experiment, by fixing the light flux at the cornea and using a constant illuminated field, a high contrast central stimulus and a nearly emmetropic population unable to elicite refractive changes by means of accommodation. 
The average proximal miosis from $3.0 \mathrm{~m}$ to $0.33 \mathrm{~m}$ was $-0.23 \mathrm{~mm} / \mathrm{D}$ (range $-0.53 \mathrm{~mm} / \mathrm{D}$ to $+0.08 \mathrm{~mm} / \mathrm{D}$ ). For a similar range of stimulus distances, Schafer and Weale [27] reported a slightly stronger miosis $(-0.33 \mathrm{~mm} / \mathrm{D})$ in a small group of presbyopes, while Chateau et al. [2], in a larger group, found a value of $-0.50 \mathrm{~mm} / \mathrm{D}$, for object distances between $4.5 \mathrm{~m}$ and $0.40 \mathrm{~m}$. These differences in pupil miosis may be associated to variations in the viewing conditions between the stimulus at different distances. For instance, in the Chateau et al. study [2] the stimulus luminance was controlled but the field size was determined by the size of distance and near VA charts, which may change the amount of light reaching the cornea. Almutairi et al. [24], using more controlled light conditions, found a rate of proximal miosis of $-0.24 \pm 0.19 \mathrm{~mm} / \mathrm{D}$ (range: -0.56 to $0.20 \mathrm{~mm} / \mathrm{D}$ ), similar to that found in the present study.

Age is a well-known factor influencing the pupil size [50,51]. In the present study, significant correlation was found between maximum pupil size and age (Pearson: $R=-0.27, p=0.047$ ), with individuals on their 50s showing an average pupil of $5.0 \mathrm{~mm}$ and individuals in their $80 \mathrm{~s}$ presenting a $4.1 \mathrm{~mm}$ average pupil. This indicates a $-0.23 \mathrm{~mm}$ decrease in pupil size per decade. This value is smaller than those reported in previous studies $(-0.4 \mathrm{~mm}$ per decade) $[20,26]$, but this difference may be fundamentally related to the age range differences in the different studies. The pupil-age variation in the Winn et al. (1994) study for the same age interval is $-0.17 \mathrm{~mm}$ per decade, which is a value closer to that found in the present pseudophakic population. The relevance of age group in the pupil diameter was recently pointed out by Guillon and colleagues [49], by reporting a smaller pupil sizes in a presbyopic population compared to pre-presbyopic subjects. In practical terms, the performance of optical components within the optical system of the eye, as is the case of the pupil, should be studied using age-matched parameters. No significant association was found between pupil miosis and age or pupil miosis and distance pupil size, indicating that in pseudophakia there was no evidence of a stronger miosis in younger individuals or individuals with larger pupil sizes, similar to observations done in phakic eyes [23,24].

As far as the authors are aware, the pupil variation as function of object vergence presented are the first models describing the pupil behavior with proximal objects in a pseudophakic population. Both models give a good description of the pupil size variability as function of object position $\left(R^{2}=0.985\right)$. Introducing age and inevitably the interaction age and proximal miosis, does not improve significantly the prediction ability of the model, but accounts for the effect of age on pupil size and therefore could be used when pupil size predictions need to be made for the extreme age ranges. The linear model chosen provided a good description of the pupil size variation for the object range studied in the present study. A more complex model, for instance using a sigmoidal function, may be necessary to account for the plateaus observed in pupil dynamics for light response [28] and accommodation [45]. The lack of stimulus in distances further than $3 \mathrm{~m}$ and closer than $0.33 \mathrm{~m}$ prevented from fitting a more elaborated model. Nevertheless, the distances included in the present study resemble the most commonly studied viewing conditions in clinics and are a good approximation to real life conditions. When age was introduced in the regression mixed effects model, a slightly smaller value of $-0.22 \mathrm{~mm}$ decrease per decade was obtained due to a small contribution of age slope imparted to interaction of age and object vergence.

Table 5. Previously published results for (mean and 95\% CIs) VA estimation (logMAR) for different viewing distances. The results for the present study are added, with simulated bounds computed from the regression model single prediction $95 \%$ CIs as explained in the text.

\begin{tabular}{lccc}
\hline Study & Distance 6.0 m & Intermediate 1.0 m & Near 0.33 m \\
\hline Shentu et al., 2001 [52] & $+0.04(-0.08,0.10)$ & - & $+0.61(0.34,0.74)$ \\
Pieh et al., 2002 [53] & $+0.10(-0.06,0.18)$ & $+0.16(-0.11,0.29)$ & $+0.58(0.34,0.70)$ \\
Rocha et al., 2007 [54] & $+0.03(-0.05,0.07)$ & $+0.33(0.04,0.47)$ & $+0.39(0.06,0.55)$ \\
Navavaty et al., 2009 [55] & $-0.02(-0.22,0.08)$ & - & $+0.57(0.28,0.71)$ \\
Present study & $-0.03(-0.10,0.05)$ & $+0.07(0.00,0.11)$ & $+0.34(0.27,0.42)$ \\
\hline
\end{tabular}


Optical modeling of the visual performance, expressed by the inverse area under the MTF, of pseudophakic eyes for a range of object vergences showed a nearly linear decrease in retinal image quality from far to near stimuli (see Figure 4). Transformation of area under the MTF to logMAR VA (MAR: minimum angle of resolution) [38], shows a decrease in VA, similar to clinical measurements [53]. Visual acuity estimation for the average distance pupil (4.45 $\mathrm{mm})$ and mean proximal miosis $(-0.23 \mathrm{~mm} / \mathrm{D})$ changed from -0.03 to $+0.34 \log$ MAR when object position varied from 3.0 to $0.33 \mathrm{~m}$.

This VA variation from a distant to a near object resembles those reported in pseudophakic patients implanted with spherical IOLs, however the VA predictions reported in this study tend to be better than those reported (Table 5). This effect may be related to the weight of central and peripheral rays on visual function, i.e., the Stiles-Crawford effect [56]. Although the model applied in this study used pupil apodization to take into account the Stiles-Crawford effect, the weight of the myopic peripheral rays, produced by the positive spherical aberration, in creating the retinal image might have been stronger than in real eyes.

The inter-individual variability in pupil size produced variations in VA of approximately $0.15 \log$ MAR. The magnitude of the variations is nearly twice the standard deviations normally reported in VA for pseudophakic patients when observing a distant object (Table 5), thus suggesting that pupil size variability may be the main factor contributing to the variability in VA in a pseudophakic population while looking at a distant object. As the defocus level increases, by moving the object closer, clinical VA data show a much higher variability as can be seen by the larger confidence intervals in near VAs (approximately $0.4 \log$ MAR). This increase in the VA variability occurs because VA in the presence of defocus is more rapidly affected by defocus in larger pupils than in smaller pupils [57]. As argued previously, the effect of the peripheral myopic rays on the retinal image might have partially compensated the near object induced vergence, improving the image quality. In a pseudophakic emmetropic eye, the effect of pupil size on VA can be estimated as a decrease of 0.04 logMAR per mm of pupil diameter increase while looking at infinity, however, when looking at a near object, this effect may be higher. The effect of proximal miosis variability on VA modeled for a fixed distance pupil size shows a much smaller effect on the optical quality and hence on VA than the effects of intra-individual pupil size. The range of proximal miosis $(-0.04$ to $+0.53 \log$ MAR) produced a variation in near VA of $0.04 \log M A R$ for the closest object position. This value represents less than half a line of VA, which is below the clinical difference considered in VA measurements, indicating that accounting for the variability in proximal miosis has a minimal effect in explaining clinical variations in VA.

The authors acknowledge that using a single stimulus luminance level is a limitation of this study. Pupil behavior is mainly driven by the response of the amount of light reaching the corneal plane [28], thus investigating the proximal miosis behavior in the presence of various light levels would have added information on how pupil miosis varies in different lighting conditions. Guillon et al. [49] reported significant interactions in pupil size measurements when these were done for different luminance levels, age ranges and refractive error. Essentially, low levels of luminance allow for more interactions between pupil size and factors influencing it due to the wider pupil diameter. Following the same assumptions, pupil miosis will probably exhibit the same behavior. The authors opted to choose the reported level of luminance, based on the following arguments: technically, it was the highest luminance level provided by the monitor, and the luminance level is close to the commonly recommended for VA measurements [58] and was used in Alarcon's study [38] to produce VA predictions, allowing us to discuss the effect of pupil inter-subject variability on VA and provide a model for pupil size description in standard clinical conditions.

The optical simulations revealed that the VA outcomes are very sensitive to the optimization method applied in the far distance condition. The optimization of the VSMTF for the far distance was intended to simulate the best spectacle refraction condition obtained for each pupil size that was targeted by the surgical process planning. However, as the far distance results reveal, this procedure tends to overestimate the VA, and the same trend can be found at intermediate and near distances. 
It should be noted that optimization over clinical data is not the same as optimization over a computer simulation model, in the sense that the best possible MTF curves that can be obtained in the latter case can easily be made better that in the former case. This would lead to an overestimation of optical performance by the LBME at both near and far distances. Furthermore, the conversion of the area under the MTF curve into VA using Equation (10) are based on the correlations obtained using clinical data versus an optical bench model [38] and a variety of IOL designs, while a computer model of a pseudophakic eye with a specific spherical IOL was used in this study. It would interesting, in a future work, to compare the correlations of clinical data to an existing computer eye model, or even develop a model specially tailored for pseudophakic eye studies, thus overcoming the modeling limitations found in this work.

\section{Conclusions}

In this study, the pupil diameter of pseudophakic participants was systematically assessed for objects positioned at different distances from the eye. A model based on linear regression is presented to describe the pupil variation as a function of object position. Optical modeling demonstrates that inter-individual pupil variability is responsible for some degree of variation in the predicted VA that can help to explain some of the VA variability reported in clinical studies. These findings support the importance of pupil size measurement for the prediction of visual performance in pseudophakia, and indicate that proximal miosis variability is unlikely to be a relevant factor in explaining the variability in VA. In practical terms, the models developed can be applied in visual performance modeling for the study of intraocular lenses.

Author Contributions: The work described in this article results from the collaborative effort of all authors. Conceptualization, E.F., P.F., R.G., and P.S.; Methodology, E.F., P.F., and P.S.; Software, E.F., and P.F.; Data Curation, P.F., R.G., and P.S.; Investigation, E.F., R.G., A.S.T., A.B., and P.S.; Resources, A.S.T.; Writing-Original Draft Preparation, E.F., and P.S.; Writing—Review and editing, A.B., Visualization, E.F., and P.F., and Supervision, P.S.

Funding: This research was partially supported by FCT-Fundação para a Ciência e a Tecnologia (within project Pest-OE/CTM/UI0195/2014).

Acknowledgments: The authors would like to acknowledge Jose Ramon Gaspar for his assistance in data collection and the Clinical and Experimental Centre of Optometry (University of Beira Interior) for using the binocular eye-tracker. The authors would also like to acknowledge Lambda Research's donation of OSLO Premium under the University License Program.

Conflicts of Interest: The authors declare no conflict of interest.

\section{References}

1. Burd, H.J.; Judge, S.J.; Flavell, M.J. Mechanics of accommodation of the human eye. Vis. Res. 1999, 39, 1591-1595. [CrossRef]

2. Chateau, N.; De, J.B.; Bouchard, F.; Molenaar, H. Infrared pupillometry in presbyopes fitted with soft contact lenses. Optom. Vis. Sci. 1996, 73, 733-741. [CrossRef]

3. Marchini, G.; Pedrotti, E.; Modesti, M.; Visentin, S.; Tosi, R. Anterior segment changes during accommodation in eyes with a monofocal intraocular lens: High-frequency ultrasound study. J. Cataract Refract. Surg. 2008, 34, 949-956. [CrossRef]

4. Modesti, M.; Pasqualitto, G.; Appolloni, R.; Pecorella, I.; Sourdille, P. Preoperative and postoperative size and movements of the lens capsular bag: Ultrasound biomicroscopy analysis. J. Cataract Refract. Surg. 2011, 37, 1775-1784. [CrossRef]

5. Findl, O.; Kiss, B.; Petternel, V.; Menapace, R.; Georgopoulos, M.; Rainer, G.; Drexler, W. Intraocular lens movement caused by ciliary muscle contraction. J. Cataract Refract. Surg. 2003, 29, 669-676. [CrossRef]

6. Marcos, S.; Ortiz, S.; Pérez-Merino, P.; Birkenfeld, J.; Durán, S.; Jiménez-Alfaro, I. Three-dimensional evaluation of accommodating intraocular lens shift and alignment in vivo. Ophthalmology 2014, 121, 45-55. [CrossRef] 
7. Elder, M.J.; Murphy, C.; Sanderson, G.F. Apparent accommodation and depth of field in pseudophakia. J. Cataract Refract. Surg. 1996, 22, 615-619. [CrossRef]

8. Langenbucher, A.; Seitz, B.; Huber, S.; Nguyen, N.X.; Küchle, M. Theoretical and measured pseudophakic accommodation after implantation of a new accommodative posterior chamber intraocular lens. Arch. Ophthalmol. 2003, 121, 1722-1727. [CrossRef]

9. Nakazawa, M.; Ohtsuki, K. Apparent accommodation in pseudophakic eyes after implantation of posterior chamber intraocular lenses. Am. J. Ophthalmol. 1983, 96, 435-438. [CrossRef]

10. Nakazawa, M.; Ohtsuki, K. Apparent accommodation in pseudophakic eyes after implantation of posterior chamber intraocular lenses: Optical analysis. Investig. Ophthalmol. Vis. Sci. 1984, 25, 1458-1460. [CrossRef]

11. Hayashi, K.; Hayashi, H.; Nakao, F.; Hayashi, F. Aging changes in apparent accommodation in eyes with a monofocal intraocular lens. Am. J. Ophthalmol. 2003, 135, 432-436. [CrossRef]

12. Trindade, F.; Oliveira, A.; Frasson, M. Benefit of against-the-rule astigmatism to uncorrected near acuity. J. Cataract Refract. Surg. 1997, 23, 82-85. [CrossRef]

13. Oshika, T.; Mimura, T.; Tanaka, S.; Amano, S.; Fukuyama, M.; Yoshitomi, F.; Maeda, N.; Fujikado, T.; Hirohara, Y.; Mihashi, T. Apparent accommodation and corneal wavefront aberration in pseudophakic eyes. Investig. Ophthalmol. Vis. Sci. 2002, 43, 2882-2886.

14. Nishi, T.; Nawa, Y.; Ueda, T.; Masuda, K.; Taketani, F.; Hara, Y. Effect of total higher-order aberrations on accommodation in pseudophakic eyes. J. Cataract Refract. Surg. 2006, 32, 1643-1649. [CrossRef]

15. Fukuyama, M.; Oshika, T.; Amano, S.; Yoshitomi, F. Relationship between apparent accommodation and corneal multifocality in pseudophakic eyes. Ophthalmology 1999, 106, 1178-1181. [CrossRef]

16. Kamiya, K.; Kawamorita, T.; Uozato, H.; Kasugai, H.; Shimizu, K. Effect of astigmatism on apparent accommodation in pseudophakic eyes. Optom. Vis. Sci. 2012, 89, 148-154. [CrossRef]

17. Serra, P.N. The Effect of Meridional Blur in Pseudoaccommodation. Ph.D. Thesis, University of Bradford: Bradford School of Optometry and Vision Sciences, Bradford, UK, 2013.

18. Atchison, D.A.; Smith, G. Optics of the Human Eye, 1st ed.; Butterworth-Heinemann: Edinburgh, UK, 2000.

19. Kasthurirangan, S.; Glasser, A. Age related changes in the characteristics of the near pupil response. Vis. Res. 2006, 46, 1393-1403. [CrossRef]

20. Winn, B.; Whitaker, D.; Elliott, D.B.; Phillips, N.J. Factors affecting light-adapted pupil size in normal human subjects. Investig. Ophthalmol. Vis. Sci. 1994, 35, 1132-1137.

21. Artigas, J.M.; Menezo, J.L.; Peris, C.; Felipe, A.; Díaz-Llopis, M. Image quality with multifocal intraocular lenses and the effect of pupil size: Comparison of refractive and hybrid refractive-diffractive designs. J. Cataract Refract. Surg. 2007, 33, 2111-2117. [CrossRef]

22. Alfonso, J.F.; Fernández-Vega, L.; Baamonde, M.B.; Montés-Micó, R. Correlation of pupil size with visual acuity and contrast sensitivity after implantation of an apodized diffractive intraocular lens. J. Cataract Refract. Surg. 2007, 33, 430-438. [CrossRef]

23. Radhakrishnan, H.; Neil Charman, W. Age-related changes in static accommodation and accommodative miosis. Ophthalmic Physiol. Opt. 2007, 27, 342-352. [CrossRef]

24. Almutairi, M.S.; Altoaimi, B.H.; Bradley, A. Accommodation and pupil behaviour of binocularly viewing early presbyopes. Ophthalmic Physiol. Opt. 2017, 37, 128-140. [CrossRef]

25. Wachler, B.S.B. Effect of pupil size on visual function under monocular and binocular conditions in LASIK and non-LASIK patients. J. Cataract Refract. Surg. 2003, 29, 275-278. [CrossRef]

26. Nakamura, K.; Bissen-Miyajima, H.; Oki, S.; Onuma, K. Pupil sizes in different Japanese age groups and the implications for intraocular lens choice. J. Cataract Refract. Surg. 2009, 35, 134-138. [CrossRef]

27. Schäfer, W.; Weale, R. The influence of age and retinal illumination on the pupillary near reflex. Vis. Res. 1970, 10, 179-191. [CrossRef]

28. Watson, A.B.; Yellott, J.I. A unified formula for light-adapted pupil size. J. Vis. 2012, 12, 12. [CrossRef]

29. Fonseca, E.S.; Fiadeiro, P.T.; Gomes, R.; Trancon, A.S.; Baptista, A.M.; Serra, P. Influence of pupil function in pseudophakia. In Proceedings of the Fourth International Conference on Applications of Optics and Photonics, Lisbon, Portugal, 31 May-4 June 2019; Volume 11207, p. 112071N.

30. Lindstrom, M.J.; Bates, D.M. Nonlinear mixed effects models for repeated measures data. Biometrics 1990, 46, 673-687. [CrossRef] 
31. Pinheiro, J.C.; Bates, D.M.; Lindstrom, M.J. Model Building for Nonlinear Mixed Effects Models; University of Wisconsin, Department of Biostatistics Madison: Madison, WI, USA, 1995.

32. Akaike, H. A new look at the statistical model identification. In Selected Papers of Hirotugu Akaike; Springer: New York, NY, USA, 1974; pp. 215-222.

33. Schwarz, G. Estimating the dimension of a model. Ann. Stat. 1978, 6, 461-464. [CrossRef]

34. Liou, H.L.; Brennan, N.A. Anatomically accurate, finite model eye for optical modeling. J. Opt. Soc. Am. A 1997, 14, 1684-1695. [CrossRef]

35. Eppig, T.; Scholz, K.; Löffler, A.; Meßner, A.; Langenbucher, A. Effect of decentration and tilt on the image quality of aspheric intraocular lens designs in a model eye. J. Cataract Refract. Surg. 2009, 35, 1091-1100. [CrossRef]

36. Thibos, L.N.; Hong, X.; Bradley, A.; Applegate, R.A. Accuracy and precision of objective refraction from wavefront aberrations. J. Vis. 2004, 4, 325-351. [CrossRef]

37. Campbell, F.; Green, D. Optical and retinal factors affecting visual resolution. J. Physiol. 1965, 181, 576-593. [CrossRef]

38. Alarcon, A.; Canovas, C.; Rosen, R.; Weeber, H.; Tsai, L.; Hileman, K.; Piers, P. Preclinical metrics to predict through-focus visual acuity for pseudophakic patients. Biomed. Opt. Express 2016, 7, 1877-1888. [CrossRef]

39. Thibos, L.N.; Applegate, R.A.; Schwiegerling, J.T.; Webb, R. Standards for reporting the optical aberrations of eyes. J. Refract. Surg. 2002, 18, S652-S660.

40. MacRae, S.; Holladay, J.T.; Glasser, A.; Calogero, D.; Hilmantel, G.; Masket, S.; Stark, W.; Tarver, M.E.; Nguyen, T.; Eydelman, M. Special report: American academy of ophthalmology task force consensus statement for extended depth of focus intraocular lenses. Ophthalmology 2017, 124, 139-141. [CrossRef]

41. Xu, R.; Wang, H.; Jaskulski, M.; Kollbaum, P.; Bradley, A. Small-pupil versus multifocal strategies for expanding depth of focus of presbyopic eyes. J. Cataract Refract. Surg. 2019, 45, 647-655. [CrossRef]

42. Xu, R.; Wang, H.; Thibos, L.N.; Bradley, A. Interaction of aberrations, diffraction, and quantal fluctuations determine the impact of pupil size on visual quality. J. Opt. Soc. Am. A 2017, 34, 481-492. [CrossRef]

43. Hess, E.H.; Polt, J.M. Pupil size in relation to mental activity during simple problem-solving. Science 1964, 143, 1190-1192. [CrossRef]

44. Hoeks, B.; Levelt, W.J. Pupillary dilation as a measure of attention: A quantitative system analysis. Behav. Res. Methods Instrum. Comput. 1993, 25, 16-26. [CrossRef]

45. Kasthurirangan, S.; Glasser, A. Characteristics of pupil responses during far-to-near and near-to-far accommodation. Ophthalmic Physiol. Opt. 2005, 25, 328-339. [CrossRef]

46. Barbur, J.L. Learning from the pupil-studies of basic mechanisms and clinical applications. Vis. Neurosci. 2004, 1, 641-656.

47. Crawford, B. The dependence of pupil size upon external light stimulus under static and variable conditions. Proc. R. Soc. Lond. Ser. B Biol. Sci. 1936, 121, 376-395.

48. Atchison, D.A.; Girgenti, C.C.; Campbell, G.M.; Dodds, J.P.; Byrnes, T.M.; Zele, A.J. Influence of field size on pupil diameter under photopic and mesopic light levels. Clin. Exp. Optom. 2011, 94, 545-548. [CrossRef]

49. Guillon, M.; Dumbleton, K.; Theodoratos, P.; Gobbe, M.; Wooley, C.B.; Moody, K. The effects of age, refractive status, and luminance on pupil size. Optom. Vis. Sci. 2016, 93, 1093. [CrossRef]

50. Birren, J.E.; Casperson, R.C.; Botwinick, J. Age changes in pupil size. J. Gerontol. 1950, 5, 216-221. [CrossRef]

51. Kumnick, L.S. Aging and the efficiency of the pupillary mechanism. J. Gerontol. 1956, 11, 160-164. [CrossRef]

52. Shentu, X.; Tang, X.; Yao, K. Spherical aberration, visual performance and pseudoaccommodation of eyes implanted with different aspheric intraocular lens. Clin. Exp. Ophthalmol. 2008, 36, 620-624. [CrossRef]

53. Pieh, S.; Kellner, C.; Hanselmayer, G.; Lackner, B.; Schmidinger, G.; Walkow, T.; Sticker, M.; Weghaupt, H.; Fercher, A.F.; Skorpik, C. Comparison of visual acuities at different distances and defocus curves. J. Cataract Refract. Surg. 2002, 28, 1964-1967. [CrossRef]

54. Rocha, K.M.; Soriano, E.S.; Chamon, W.; Chalita, M.R.; Nosé, W. Spherical aberration and depth of focus in eyes implanted with aspheric and spherical intraocular lenses: A prospective randomized study. Ophthalmology 2007, 114, 2050-2054. [CrossRef]

55. Nanavaty, M.A.; Spalton, D.J.; Boyce, J.; Saha, S.; Marshall, J. Wavefront aberrations, depth of focus, and contrast sensitivity with aspheric and spherical intraocular lenses: Fellow-eye study. J. Cataract Refract. Surg. 2009, 35, 663-671. [CrossRef]

56. Moon, P.; Spencer, D.E. On the stiles-crawford effect. J. Opt. Soc. Am. 1944, 34, 319-329. [CrossRef] 
57. Atchison, D.A.; Smith, G.; Efron, N. The effect of pupil size on visual acuity in uncorrected and corrected myopia. Am. J. Optom. Physiol. Opt. 1979, 56, 315-323. [CrossRef]

58. Sheedy, J.E.; Bailey, I.L.; Raasch, T.W. Visual acuity and chart luminance. Am. J. Optom. Physiol. Opt. 1984, 61, 595-600. [CrossRef]

(C) 2019 by the authors. Licensee MDPI, Basel, Switzerland. This article is an open access article distributed under the terms and conditions of the Creative Commons Attribution (CC BY) license (http:/ / creativecommons.org/licenses/by/4.0/). 